\title{
Dark matter searches in XMASS
}

\section{Kazuyoshi Kobayashi*}

Kamioka observatory, Institute of Cosmic Ray Research, the University of Tokyo, Higashi-Mozumi, Kamioka, Hida, Gifu, 506-1205, Japan.

Kavli Institute for the Physics and Mathematics of the Universe (WPI), the University of Tokyo, Kashiwa, Chiba, 277-8582, Japan.

E-mail: kenkoudicrr.u-tokyo.ac.jp

With $835 \mathrm{~kg}$ liquid xenon, XMASS searched for various kinds of dark matter candidate. From November 2013, we have been taking data for more than 4.5 years. We report standard WIMP, annual modulation, hidden photon and axion-like particle searches. No significant signals are observed in these searches.

The 39th International Conference on High Energy Physics

4-11 July 2018

Seuol, South Korea

${ }^{*}$ Speaker. 


\section{Introduction}

The XMASS project aims at detecting dark matter, pp and 7Be solar neutrinos, and neutrino less double beta decay using large volume of pure liquid xenon. The first physics target of the XMASS experiment is to detect dark matter. Various astronomical observations provide strong evidence for a large amount of dark matter in the universe. However, its nature is still unknown. Weakly interacting massive particles (WIMPs) are dark matter candidates motivated by theories beyond the Standard Model. Although many direct dark matter search experiments have searched for WIMPs, dark matter have not been observed yet. This project searches for nuclear recoils in liquid xenon caused by WIMPs. Since XMASS is sensitive not only to nuclear recoils but also electron recoils, various candidates of dark matter are also searched for.

\section{XMASS detector}

The XMASS detector is located in the Kamioka mine $1000 \mathrm{~m}$ underneath the top of Mt. Ikenoyama (i.e. $2700 \mathrm{~m}$ water equivalent underground) in Japan. The detector consists of two components, the inner and outer detectors (ID and OD, respectively). The ID is equipped with 642 inward-facing photomultiplier tubes (PMTs) in an approximate spherical shape in a copper vessel filled with pure liquid xenon. The entire structure is immersed in liquid xenon. The amount of liquid xenon in the sensitive region is $835 \mathrm{~kg}$. The PMT photo coverage of more than $62 \%$ is achieved and the PMT quantum efficiency at the scintillation wavelength of liquid xenon $(175 \mathrm{~nm})$ is more than $28 \%$. The vessel which holds liquid xenon and the PMT holder is made of oxygen free high conductivity copper (OFHC) and the size is $1120 \mathrm{~mm}$ in diameter. To reduce the amount of liquid xenon, an OFHC copper filler is installed in the gap between the PMT holder and the inner vessel. The vessel is covered with another vessel for vacuum insulation. The ID is installed at the centre of the OD, which is a cylindrical water tank (10 $\mathrm{m}$ in height and $10 \mathrm{~m}$ in diameter) with seventy two 20-inch PMTs. The OD is used as an active shield for cosmic ray muons and a passive shield for low-energy gamma rays and neutrons. After the detector construction, commissioning runs were conducted from October 2010 to June 2012. Data taking started November 2013 After the detector refurbishment and continues. We have already taken data for more than 4.5 years.

\section{Physics results from XMASS}

We are using for various methods to search for dark matters. We report three of them, that are standard WIMP search, annual modulation search, and hidden photon/axion-like particle search.

\subsection{Standard WIMP search}

We have searched for standard WIMP, which is our primary aim to detect, using 705.9 live days of data in a fiducial volume containing $97 \mathrm{~kg}$ of liquid xenon at the center of the detector. We uses the events after passing through our standard cut, fiducial volume cut using photo electron based and timing based vertex reconstruction. The events with reconstructed energy more than $2 \mathrm{keV}_{e e}$ are used. The event rate in the fiducial volume after the data reduction was $(4.2 \pm 0.2) \times 10^{-3} \mathrm{day}^{-1} \mathrm{~kg}^{-1} \mathrm{keV}_{e e}^{-1}$ at $5 \mathrm{keV}_{e e}$, with a signal efficiency of $20 \%$. All the remaining 
events are consistent with our background evaluation based on independent assays of background radioactivities and simultaneous fitting of signal and background, shown in Figure $\mathrm{W}$. Most of backgrounds come from the mis-reconstructed events originated from ${ }^{210} \mathrm{~Pb}$ in the copper plates lining the detector's inner surface. In the study of ${ }^{210} \mathrm{~Pb}$ in copper [व], we found that even OFHC contain a few tens $\mathrm{mBq} / \mathrm{kg}$ of ${ }^{210} \mathrm{~Pb}$ in bulk. In the OFHC in XMASS, ${ }^{210} \mathrm{~Pb}$ concentration is estimated to be $25 \pm 5 \mathrm{mBq} / \mathrm{kg}$, that is consistent to the measurement. Figure $\square$ shows the spin-independent WIMP nucleon cross section limit. The obtained limit was $2.2 \times 10^{44} \mathrm{~cm}^{2}$ for a WIMP mass of 60 $\mathrm{GeV} / \mathrm{c}^{2}$ at the $90 \%$ confidence level (CL), which was the most stringent limit among results from single-phase liquid xenon detectors [3]].

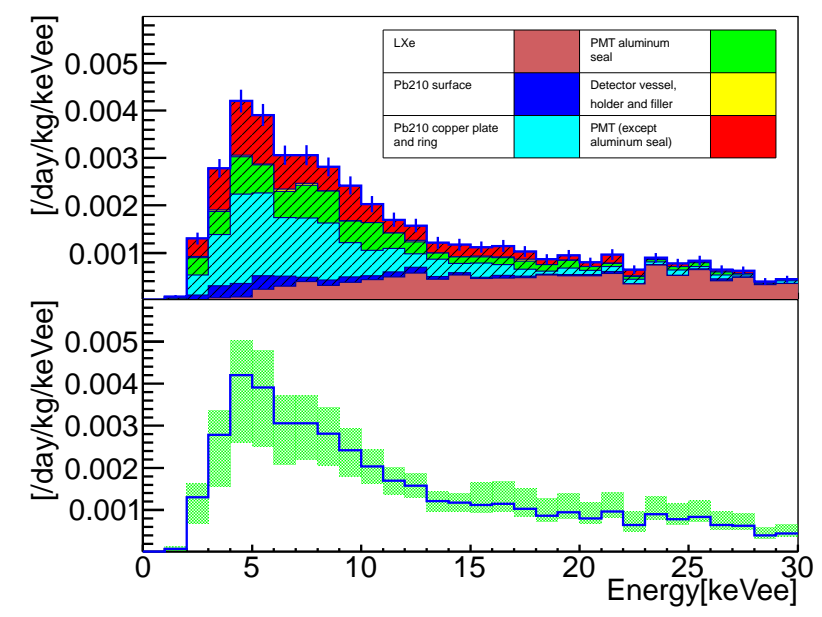

Figure 1: Expected background from MC study. Upper figure shows the breakdown. Lower figure shows total expected background (blue line) with systematic errors (green region).

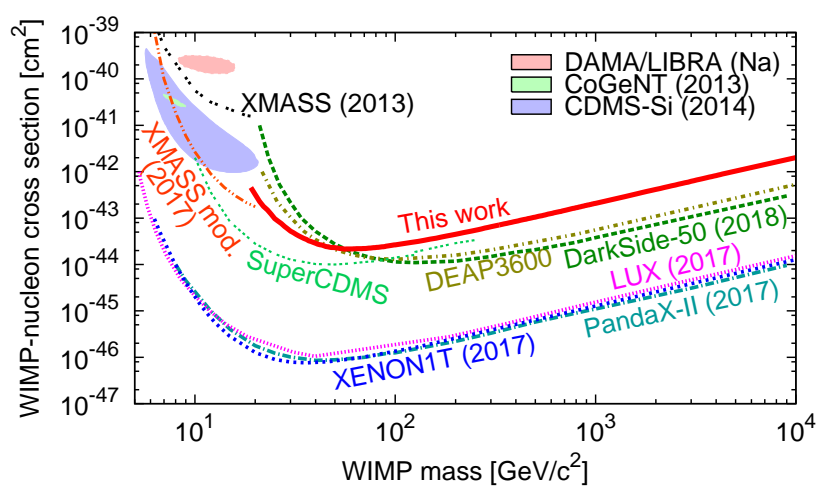

Figure 2: The spin-independent WIMP-nucleon cross section limit as a function of theWIMP mass at the $90 \%$ CL. The XMASS limit is shown by the solid line compared with other experimental results.

\subsection{Annual modulation search}

An annual modulation signal coming from the earth orbiting around the sun would be one of 
the indications of dark matter signal. We use 800 live days of data with $832 \mathrm{~kg}$ target mass to search. Simple event selection is performed instead of vertex reconstruction in order to use lower energy events than that of standar WIMP search. But no sigificant signal was found. Assuming WIMP dark matter elastically scattering on the xenon target, the exclusion upper limit for the WIMPnucleon cross section was $1.9 \times 10^{41} \mathrm{~cm}^{2}$ at $8 \mathrm{GeV} / \mathrm{c}^{2}$ at $90 \% \mathrm{CL}$. For the model-independent case, without assuming any specific dark matter model, we obtained consistent result with the null hypothesis with a p-value of 0.11 in the $1-20 \mathrm{keV}$ energy region. This search probed this region with an exposure that was larger than that of DAMA/LIBRA. We also did not find any significant amplitude in the data for periodicity with periods between 50 and 600 days in the energy region between 1 to $6 \mathrm{keV}$ [四].

\subsection{Hidden photon and axion-like particle search}

Hidden photons and axion-like particles are candidates for cold dark matter if they were produced non-thermally in the early universe. We conducted a search for both of these bosons using 800 live days of data with $327 \mathrm{~kg}$ of liquid xenon in the fiducial volume. Expected peak in number of photo electron distribution is searched for in 590-1760 PE region which corresponding to 40-120 $\mathrm{keV}$. No significant signal was observed, and thus we set constraints on the $\alpha^{\prime} / \alpha$ parameter related to kinetic mixing of hidden photons and the coupling constant $\mathrm{g}_{A e}$ of axion-like particles in the mass range from 40 to $120 \mathrm{keV} / c^{2}$, resulting in $\alpha^{\prime} / \alpha<6 \times 10^{26}$ and $\mathrm{g}_{A e}<4 \times 10^{13}$. These limits are the most stringent derived from both direct and indirect searches to date. The detail is shown in Ref. [[]].

\section{Summary}

XMASS have been taking data more than 4.5 years since November 2013. Three kinds of dark matter search using the XMASS data are shown. No significant signal of dark matters are found. We are analyzing data for other dark matter searches and other physics topics as well. We keep searching for dark matter in XMASS.

\section{References}

[1] K. Abe et al. (XMASS collaboration), XMASS detector, Nucl. Instr. and Meth. A 716, (2013) 78-85 [arXiv:1301.2815].

[2] K. Abe et al. (XMASS collaboration), Identification of ${ }^{210} \mathrm{~Pb}$ and ${ }^{210} \mathrm{Po}$ in the bulk copper samples with a low-background alpha particle counter, Nucl. Instr. and Meth. A 884, (2018) 157-161 [arXiv:1707.06413].

[3] K. Abe et al. (XMASS collaboration), A direct dark matter search in XMASS-I, Phys. Lett.B accepted [arXiv:1804.02180].

[4] K. Abe et al. (XMASS collaboration), Direct dark matter search by annual modulation with 2.7 years of XMASS-I data, Phys. Rev. D97, 102006 (2018) [arXiv: 1801.10096 ].

[5] K. Abe et al. (XMASS collaboration), Search for dark matter in the form of hidden photons and axion-like particles in the XMASS detector, Phys. Lett.B 787, (2018) 153-158 [arXiv:1807.08516]. 